\title{
Quantum Kinetic Equations and Dark Matter Abundances Reconsidered
}

\author{
Anupam Singh* \\ Theoretical Division, T-8, Los Alamos National Laboratory, Los Alamos, NM 87545 \\ Mark Srednicki ${ }^{\dagger}$ \\ Department of Physics, University of California, Santa Barbara, CA 93106
}

\begin{abstract}
Starting from a Caldeira-Leggett model for the interaction of a system with an environment, Joichi, Matsumoto, and Yoshimura have reconsidered the derivation of the quantum Boltzmann equation. They find an extra term that accounts for the effects of virtual particles, and which drastically changes the results for relic densities of stable, weakly interacting massive particles (WIMPs), and for the decay products of unstable particles. We show, however, that this modified Boltzmann equation does not properly account for the interaction energy between the massive particles (which are decaying or annihilating) and the thermal bath of light particles. We argue that the conventional Boltzmann equation gives the correct result.
\end{abstract}

${ }^{*}$ E-mail: singh@lanl.gov

$\dagger$ E-mail: mark@physics.ucsb.edu 
Joichi, Matsumoto, and Yoshimura (hereafter JMY) [1.2] and Matsumoto and Yoshimura (hereafter MY) [3] have carefully reconsidered the derivation of the quantum Boltzmann equation for heavy particles embedded in a thermal bath of light particles. JMY treat the case of unstable heavy particles, and MY treat the case of a stable, weakly interacting massive particles (WIMPs) that annihilate to light particles. In both cases they find new terms in the quantum Boltzmann equation that account for the effects of virtual heavy particles, and that drastically change the usual formula for the equilibrium abundance of these particles. In particular, the usual calculation of relic abundances of WIMPs is completely changed, with the result that a WIMP with a mass in excess of approximately $1 \mathrm{GeV}$ is would overclose the universe, for a broad range of interaction strengths with the light particles.

These surprising results must be taken seriously, since previous derivations of the quantum Boltzmann equation (for weakly interacting massive particles) can involve uncontrolled approximations and possibly arguable assumptions (see, e.g., [4] for a typical treatment). In this context the analyses of JMY and MY are among the most rigorous ones available.

The basic issue raised by JMY is more easily understood in the context of an unstable, decaying particle (rather than stable, annihilating particles). Given a spin-zero particle $\varphi$ with mass $M$ and energy $E(\mathbf{p})=\left(\mathbf{p}^{2}+M^{2}\right)^{1 / 2}$ at a temperature $T$, the conventional formula for its equilibrium number density is

$$
\begin{aligned}
n_{\varphi} & =\int \frac{d^{3} p}{(2 \pi)^{3}} \frac{1}{e^{E(\mathbf{p}) / T}-1} \\
& = \begin{cases}\zeta(3) T^{3} / \pi^{2} & \text { for } T \gg M, \\
(M T / 2 \pi)^{3 / 2} e^{-M / T} & \text { for } T \ll M .\end{cases}
\end{aligned}
$$

JMY, on the other hand, argue that if the particle is weakly coupled, and unstable with a decay width $\Gamma \ll M$, then we should have instead [2]

$$
n_{\varphi}=\int \frac{d^{3} p}{(2 \pi)^{3}} \int_{p}^{\infty} d \omega \frac{\Gamma / 2 \pi}{(\omega-E(\mathbf{p}))^{2}+(\Gamma / 2)^{2}} \frac{1}{e^{\omega / T}-1} .
$$

That is, we should allow the energy of the unstable particle to vary according to a BreitWigner function, rather than be fixed at $\omega=E(\mathbf{p})$. (For simplicity of notation, we have left out a time-dilation factor of $M / E(\mathbf{p})$ that should multiply $\Gamma$; this will not affect our subsequent analysis, which is primarily concerned with the nonrelativistic regime.) If we take the limit $\Gamma \rightarrow 0$, then the Breit-Wigner function becomes $\delta(\omega-E(\mathbf{p}))$, and we recover Eq. (11). On the other hand, if we take $T \ll \Gamma \ll M$, then the integral is dominated by the region near $\omega \sim T$, and we have instead [2]

$$
\begin{aligned}
n_{\varphi} & =\frac{\Gamma}{4 \pi^{3} M^{2}} \int_{0}^{\infty} d \omega \frac{1}{e^{\omega / T}-1} \int_{0}^{\omega} d p p^{2} \\
& =\frac{\pi}{180} \frac{\Gamma}{M^{2}} T^{4} \quad \text { for } T \ll \Gamma \ll M .
\end{aligned}
$$

This is drastically different than the usual result, Eq. (看); in particular, there is no exponential Boltzmann suppression. We see that this is essentially because the $\varphi$ particles that are being counted in Eq. (3) are far off shell, with energy near zero.

While Eq. (2) may seem plausible, it leads to some surprising conclusions. Let us assume (following [1.2]) that the $\varphi$ particle decays into two massless spin-zero $\chi$ particles via an 
interaction $\mathcal{H}_{\text {int }}=\frac{1}{2} \mu \varphi \chi^{2}$; thus we have $\Gamma=\mu^{2} / 32 \pi M$. Now suppose that we place a hot gas of light $\chi$ particles in a large box, at a temperature $T \ll M$. The number density of $\chi$ particles is $n_{\chi}=\zeta(3) T^{3} / \pi^{2}$ and their energy density is $\rho_{\chi}=\pi^{2} T^{4} / 30$. After thermal equilibrium is established between $\varphi$ and $\chi$ particles, there should be a number density $n_{\varphi}$ of $\varphi$ particles given by Eq. (3). The corresponding energy density $\rho_{\varphi}$ is obtained by including an extra factor of $E(\mathbf{p})$ in the integrand of Eq. (2) (and not, as one might guess, an extra factor of $\omega)$. For $T \ll M$, this implies

$$
\rho_{\varphi}=M n_{\varphi} \sim \Gamma T^{4} / M
$$

We now see that the ratio of $\varphi$ energy density to $\chi$ energy density is independent of temperature:

$$
\rho_{\varphi} / \rho_{\chi} \sim \Gamma / M
$$

This strikes us as odd, since there would not seem to be a source of virtual heavy $\varphi$ particles in the limit of zero temperature for the massless $\chi$ particles.

The situation worsens for the case of stable, annihilating $\varphi$ particles treated by MY [3]. For an interaction of the form $\mathcal{H}_{\text {int }}=\frac{1}{2} \lambda \varphi^{2} \chi^{2}$, MY find

$$
n_{\varphi} \sim \lambda(T / M)^{1 / 2} T^{3}
$$

This implies $\rho_{\varphi} \sim \lambda(M T)^{1 / 2} T^{3}$, and hence

$$
\rho_{\varphi} / \rho_{\chi} \sim \lambda(M / T)^{1 / 2}
$$

Thus, for $T \ll M$, we see that the energy density in virtual heavy $\varphi$ particles greatly exceeds the energy density in on-shell massless $\chi$ particles. (This is not ruled out by energy conservation; the original temperature of the $\chi$ gas would simply drop as the energy flows into virtual $\varphi$ particles.) Eq. (7) would seem to imply that (for example) the cosmic microwave background radiation is accompanied by a much larger energy density of virtual heavy particles. We believe that this is not a tenable proposition.

Where, then, is the flaw in the MY analysis? Consider a system coupled to an environment via an interaction,

$$
H=H_{\mathrm{sys}}+H_{\mathrm{env}}+H_{\mathrm{int}}
$$

where we assume that $H_{\text {sys }}$ and $H_{\text {env }}$ are positive semidefinite operators. We wish to determine the energy of the system when it is in thermal equilibrium with the environment. The most obvious candidate for this energy is

$$
E_{\mathrm{sys}}=\left\langle H_{\mathrm{sys}}\right\rangle_{T}
$$

where the angle brackets denote canonical thermal averaging with subtraction of the zeropoint energy,

$$
\langle\ldots\rangle_{T}=\frac{\operatorname{Tr} \ldots e^{-H / T}}{\operatorname{Tr} e^{-H / T}}-\langle 0|\ldots| 0\rangle
$$


This definition of $E_{\mathrm{sys}}$ is the one used by JMY and MY. However, it is reasonable if and only if

$$
\left|\left\langle H_{\text {int }}\right\rangle_{T}\right| \ll\left\langle H_{\text {sys }}\right\rangle_{T}
$$

If Eq. (11) does not hold, then the interaction between system and environment is effectively strong (no matter how small the coupling may be), and the appropriate division between system and environment is unclear.

The analyses of JMY and MY are based on consideration of a Caldeira-Leggett model [5] of coupled harmonic oscillators, grouped into terms according to Eq. (8). We will show below that in this model, at low temperature and weak coupling,

$$
\left\langle H_{\mathrm{int}}\right\rangle_{T} \simeq-2\left\langle H_{\mathrm{sys}}\right\rangle_{T}
$$

We see that the negative interaction energy more than compensates for the system energy, which our qualitative arguments indicated was much too large.

To demonstrate Eq. (12), we use the model presented in [2]. A slightly different model was used in [1]; we have checked that Eq. (12) holds in the model of [1] as well. The model of [2] is

$$
\begin{aligned}
& H_{\mathrm{sys}}=E_{1} c^{\dagger} c \\
& H_{\mathrm{env}}=\int_{\omega_{c}}^{\infty} d \omega \omega b^{\dagger}(\omega) b(\omega) \\
& H_{\mathrm{int}}=\int_{\omega_{c}}^{\infty} d \omega \sqrt{\sigma(\omega)}\left[c^{\dagger} b(\omega)+b^{\dagger}(\omega) c\right] .
\end{aligned}
$$

Here $c$ and $b(\omega)$ are harmonic-oscillator operators with commutation relations $\left[c, c^{\dagger}\right]=1$ and $\left[b(\omega), b^{\dagger}\left(\omega^{\prime}\right)\right]=\delta\left(\omega-\omega^{\prime}\right), \sigma(\omega)$ is a frequency-dependent coupling, and $\omega_{c}$ is a lower cutoff; we assume $\omega_{c} \ll E_{1}$. The exact solution of this model involves changing to new variables $B(\omega)$ such that

$$
H=H_{\mathrm{sys}}+H_{\mathrm{env}}+H_{\mathrm{int}}=\int_{\omega_{c}}^{\infty} d \omega \omega B^{\dagger}(\omega) B(\omega)
$$

where $\left[B(\omega), B^{\dagger}\left(\omega^{\prime}\right)\right]=\delta\left(\omega-\omega^{\prime}\right)$, and the original operators are given in terms of the new ones via

$$
\begin{aligned}
c & =\int_{\omega_{c}}^{\infty} d \omega \sqrt{\sigma(\omega)} f(\omega) B(\omega), \\
b(\omega) & =B(\omega)+O(\sigma) .
\end{aligned}
$$

Here the function $f(\omega)$ is given by

$$
f(\omega)=\frac{1}{\omega-E_{1}+\Pi(\omega)+i \pi \sigma(\omega)},
$$

where

$$
\Pi(\omega)=\mathrm{P} \int_{\omega_{c}}^{\infty} d \omega^{\prime} \frac{\sigma\left(\omega^{\prime}\right)}{\omega^{\prime}-\omega} .
$$


The $O(\sigma)$ term in the formula for $b(\omega)$ will not be needed; we will treat the coupling as weak, $\sigma(\omega) \ll E_{1}$, and work to leading nontrivial order in $\sigma$. This means we can neglect $\Pi(\omega)$ compared to $E_{1}$, and treat $E_{1}$ as the renormalized single-particle energy; this point is thoroughly discussed in [1].

We now wish to compute $\left\langle H_{\text {sys }}\right\rangle_{T}$ and $\left\langle H_{\text {int }}\right\rangle_{T}$. (We can also compute $\left\langle H_{\text {env }}\right\rangle_{T}$, but the result is infinite, due to the infinite number of harmonic oscillators in the environment.) This is entirely straightforward; the formula we need is

$$
\left\langle B^{\dagger}\left(\omega^{\prime}\right) B(\omega)\right\rangle_{T}=\frac{1}{e^{\omega / T}-1} \delta\left(\omega^{\prime}-\omega\right) .
$$

Using Eqs. (13,17,19,21), we have

$$
\begin{aligned}
\left\langle H_{\mathrm{sys}}\right\rangle_{T} & =E_{1} \int_{\omega_{c}}^{\infty} d \omega^{\prime} d \omega \sqrt{\sigma\left(\omega^{\prime}\right) \sigma(\omega)} f^{*}\left(\omega^{\prime}\right) f(\omega)\left\langle B^{\dagger}\left(\omega^{\prime}\right) B(\omega)\right\rangle_{T} \\
& =E_{1} \int_{\omega_{c}}^{\infty} d \omega \sigma(\omega)|f(\omega)|^{2} \frac{1}{e^{\omega / T}-1} \\
& =E_{1} \int_{\omega_{c}}^{\infty} d \omega \frac{\sigma(\omega)}{\left(\omega-E_{1}\right)^{2}+\pi^{2} \sigma^{2}(\omega)} \frac{1}{e^{\omega / T}-1} .
\end{aligned}
$$

We see the similarity with Eq. (2). At high temperature and weak coupling, the region near $\omega \sim E_{1}$ dominates, and we have

$$
\left\langle H_{\mathrm{sys}}\right\rangle_{T} \simeq \frac{E_{1}}{e^{E_{1} / T}-1} \text { for } \sigma(\omega) \ll T \sim E_{1}
$$

This is the same result that one would obtain for a noninteracting oscillator. On the other hand, at low temperature the low- $\omega$ region dominates, and we have

$$
\left\langle H_{\mathrm{sys}}\right\rangle_{T} \simeq \frac{1}{E_{1}} \int_{\omega_{c}}^{\infty} d \omega \frac{\sigma(\omega)}{e^{\omega / T}-1} \quad \text { for } T \ll \sigma(\omega) \ll E_{1} .
$$

We now turn our attention to the interaction energy. We begin by computing

$$
\begin{aligned}
\left\langle c^{\dagger} b(\omega)\right\rangle_{T} & =\int_{\omega_{c}}^{\infty} d \omega^{\prime} \sqrt{\sigma\left(\omega^{\prime}\right)} f^{*}\left(\omega^{\prime}\right)\left\langle B^{\dagger}\left(\omega^{\prime}\right) B(\omega)\right\rangle_{T}+O(\sigma) \\
& =\sqrt{\sigma(\omega)} f^{*}(\omega) \frac{1}{e^{\omega / T}-1}+O(\sigma) .
\end{aligned}
$$

From here on we do not display the $O(\sigma)$ correction. We then have

$$
\begin{aligned}
\left\langle H_{\mathrm{int}}\right\rangle_{T} & =\int_{\omega_{c}}^{\infty} d \omega \sqrt{\sigma(\omega)}\left[\left\langle c^{\dagger} b(\omega)\right\rangle_{T}+\text { c.c. }\right] \\
& =\int_{\omega_{c}}^{\infty} d \omega \frac{2\left(\omega-E_{1}\right) \sigma(\omega)}{\left(\omega-E_{1}\right)^{2}+\pi^{2} \sigma^{2}(\omega)} \frac{1}{e^{\omega / T}-1}
\end{aligned}
$$

At high temperature and weak coupling, we get

$$
\left\langle H_{\mathrm{int}}\right\rangle_{T} \simeq \mathrm{P} \int_{\omega_{c}}^{\infty} d \omega \frac{2 \sigma(\omega)}{\omega-E_{1}} \frac{1}{e^{\omega / T}-1} \quad \text { for } \sigma(\omega) \ll T \sim E_{1} .
$$


This is smaller than $\left\langle H_{\text {sys }}\right\rangle_{T}$ due to a suppression factor of $\sigma(\omega) / E_{1}$. Thus the interaction energy is small compared to the system energy, as it should be. If, however, we consider low temperature and weak coupling, then we get

$$
\left\langle H_{\mathrm{int}}\right\rangle_{T} \simeq-\frac{2}{E_{1}} \int_{\omega_{c}}^{\infty} d \omega \frac{\sigma(\omega)}{e^{\omega / T}-1} \quad \text { for } T \ll \sigma(\omega) \ll E_{1} .
$$

Comparing with Eq. (24) gives us Eq. (12).

Clearly, then, the proper identification of the system energy becomes a key issue. We do not have a definitive resolution of this puzzle. However, we offer the following comments regarding the specific problem of WIMP relic densities.

It seems to us that the WIMP relics that survive to the present day (and constitute the nonbaryonic dark matter) must be on-shell particles. If they are to be virtual, they must be produced via processes involving the present microwave-background photons; as discussed above, we find this to be untenable. Therefore, the correct computation is, we believe, of the number density of on-shell particles. This leads us back to the usual Boltzmann equation, which assumes all particles are on-shell. In fact, among the clearest derivations of this equation is the one presented by MY, before they go on to consider virtual effects. The usual Boltzmann equation of course leads to the standard result for the relic WIMP density, which we believe is correct.

\section{ACKNOWLEDGMENTS}

We thank John Ellis, Toby Falk, and Keith Olive for collaboration on the early stages of this work. We also thank Salman Habib and Emil Mottola for helpful discussions. This work

was supported in part by the United States Department of Energy at Los Alamos National Laboratory, by the National Science Foundation through grant PHY-97-22022, and by the Institute of Geophysics and Planetary Physics through grant 920. 


\section{REFERENCES}

[1] I. Joichi, Sh. Matsumoto, and M. Yoshimura, Phys. Rev. A 57, 798 (1998), hepth/9609223; Prog. Theor. Phys. 98, 9 (1997), cond-mat/9612235.

[2] I. Joichi, Sh. Matsumoto, and M. Yoshimura, Phys. Rev. D 58, 043507 (1998), hepph/9803201.

[3] Sh. Matsumoto and M. Yoshimura, Phys. Rev. D 59, 123511 (1999), hep-ph/9811301.

[4] L. P. Kadanoff and G. Baym, Quantum Statistical Mechanics (Benjamin, New York, 1962).

[5] A. O. Caldeira and A. J. Leggett, Physica 121A, 587 (1983); Ann. Phys. (N.Y.) 149, 374 (1983). 